\title{
Erratum: A new piece of the ALS puzzle
}

\section{$\mathrm{J}$ L Cleveland}

Nat. Genet. 34, 357 (2003).

The second at-risk haplotype in Figure 1 should read AGG. 